\title{
Smoking Cessation Interventions for COPD: A Review of the Literature
}

\author{
Renata Pires-Yfantouda CPsychol AFBPsS DClinPsy DHeath Psy, \\ Gareth Absalom CPsychol DHealthPsy, and Felicity Clemens MSc
}

\author{
Introduction \\ Smoking Cessation and COPD \\ Objectives \\ Methods \\ Criteria for Considering Studies for This Review \\ Search Strategy to Identify Studies \\ Study Selection Criteria \\ Methodological Quality Assessment \\ Data Analysis \\ Description of Studies \\ Methodological Quality \\ Results \\ Different Psychosocial Interventions With Pharmacotherapy \\ Comparison By Disease Presence \\ Discussion \\ Disease Severity \\ Levels of Addiction and Intensity of Intervention \\ Effect of Healthcare Professional \\ Attendance \\ Social Support and Motivation \\ Follow-up and Biomarkers \\ Conclusions
}

The aim of this systematic review is to establish the most effective stop smoking intervention approach for smokers with COPD. The search strategy included the electronic databases MEDLINE, EMBASE, AMED, PsycINFO, DARE, Cochrane Library, and CINAHL, between January 2006 and January 2010. References of the included studies were also screened for additional papers, and further hand searches were conducted. The selection criteria included randomized controlled trials or quasi-randomized controlled trials with at least one subject group diagnosed with COPD. Two independent reviewers reviewed the included studies, using a quality assessment form developed from the selection criteria. Divergence of quality assessment scores was resolved by the 2 reviewers agreeing on a score. The 4 studies selected indicate that psychosocial interventions combined with pharmacotherapy are effective in smoking cessation at 12 months post-intervention, although the effect is not statistically significant, due to small sample size and heterogeneity between the studies (odds ratio 2.35, 95\% CI 0.25-21.74,). However, despite this medium effect size, due to a lack of universal use of pharmacotherapies in most of the studies, it makes a definitive comparison of efficacy difficult to determine. This review also shows the effectiveness of psychosocial treatment for people with or without COPD symptoms at 12 months, although the effect of disease severity is not clear. This review also highlights the difficulty of maintaining attendance at community-based locations, compared to acute or research settings. Key words: COPD; psychosocial interventions; pharmacotherapy. [Respir Care 2013;58(11):1955-1962. (C) 2013 Daedalus Enterprises] 


\section{Smoking Cessation Interventions for COPD: A Review of the Literature}

\section{Introduction}

In 2007 the World Health Organization estimated that COPD affected 210 million people, and reported that in 20053 million people had died of COPD, corresponding to $5 \%$ of all global deaths. ${ }^{1}$ In 2002, Mannino ${ }^{2}$ estimated that by 2020 this disease would be the 5th largest cause of disability-adjusted life-years worldwide. More recently the World Health Organization predicted that by 2030 this disease will be the third leading cause of death worldwide. ${ }^{1}$ Not all of these cases will be directly attributable to smoking, but smoking will be the primary cause.

In the United Kingdom nearly 900,000 people have been diagnosed with COPD, as cited by the National Health Service, 2009. ${ }^{3}$ However, the United Kingdom National Health Service (2009) estimates that the majority of people with COPD are unaware of their condition and that the real figure is approximately 3 million people in the United Kingdom, with older people more likely to develop COPD.

\section{Smoking Cessation and COPD}

The National Institute for Health and Clinical Excellence 2004 quick reference guide, Chronic Obstructive Pulmonary Disease: Management of Chronic Pulmonary Disease in Adults in Primary and Secondary Care, ${ }^{4}$ recommends that all COPD patients who smoke should be encouraged to stop at every opportunity, and recommends pharmacotherapy combined with a behavioral support program. However, while the guidelines are set out by the Department of Health ${ }^{5}$ on supporting smokers in the general population to stop smoking, no guidance is yet available on the most effective way to support this chronic disease group in stopping smoking.

Many studies have found that this group differs from the general population in ways that affect their attempts at and success in stopping smoking. Some of these differences

Dr Pires-Yfantouda is affiliated with the Department of Health Psychology, City University, London, United Kingdom. Mr Absalom is affiliated with the Lambeth Stop Smoking Service, Guy and St Thomas Trust, London, United Kingdom. Ms Clemens is affiliated with the Department of Medical Statistics, London School of Hygiene and Tropical Medicine, London, United Kingdom.

The authors have disclosed no conflicts of interest.

Supplementary material related to this paper is available at http:// www.rcjournal.com.

Correspondence: Renata Pires-Yfantouda CPsychol AFBPsS DClinPsy DHeath Psy, Department of Health Psychology, City University, Northampton Square, EC1V 0HC, London, United Kingdom. E-mail: r.pires@ city.ac.uk.

DOI: $10.4187 /$ respcare. 01923 include the type of smokers they are, ${ }^{5}$ their beliefs about how their smoking behavior relates to their COPD, ${ }^{6}$ and the effect of a chronic disease on their psychological and emotional well-being. ${ }^{7}$ These factors need to be considered when designing stop-smoking interventions.

Perception of the etiology of the illness is an important factor in the decision to quit smoking. Walters and Coleman found that patients who attribute their respiratory symptoms to smoking are 8 times (95\% CI 3.0-23.3) more likely to believe that their health will improve if they stop smoking, and 6 times (95\% CI 1.4-23.3) more likely to intend to stop smoking. ${ }^{7}$

A key factor for patients with chronic diseases is adherence to treatment and long-term maintenance of the recommended behavior changes. ${ }^{8,9}$ They attribute this to a range of factors, including patients' perception of their disease, type of treatment or medication, the quality of patient-provider communication, the social environment, and the level of anxiety and depression in patients with COPD, although estimates of prevalence vary.

A combination of these factors is associated with lower self-efficacy and impaired overall health status, such as dyspnea, and loss and grief associated with the disability of COPD. ${ }^{8,10}$ Stage et al ${ }^{11}$ reported that depression is common in COPD patients, with around $40 \%$ being affected with clinical depression. Despite these findings, it is not always possible to identify the exact cause when diagnosing depression in COPD patients, because of common symptoms present in both COPD and depression, such as fatigue and altered sleep patterns. ${ }^{8}$ It is difficult to quantify how many people with COPD continue to smoke after diagnosis. However, clinical studies of medications for COPD treatment have reported figures ranging between $38 \%$ and $43 \%$ of patients with moderately severe to severe COPD continuing to smoke. ${ }^{12,13}$

Attempts to identify the most effective approach for this group to stop smoking have been made in previous systematic reviews, and have included a combination of behavioral support and nicotine replacement therapy (NRT) or alternative pharmacotherapies such as bupropion, varenicline, and nortriptyline as the most effective successful cessation interventions in this population. However, it is not clear whether, and if so how, the intervention to support an individual with COPD should differ from that offered to smokers from the general population.

In their review of 5 studies undertaken between 1991 and 2001, Wagena et al ${ }^{14}$ concluded that combining psychosocial interventions and pharmacologic interventions resulted in better outcomes than either no intervention or psychosocial interventions alone. This latter finding of the lack of evidence to support the effectiveness of any psychosocial intervention alone for smokers with COPD was due to a lack of high-quality studies comparing a combination of interventions with no intervention. A later re- 


\section{Smoking Cessation Interventions for COPD: A Review of the Literature}

view ${ }^{15}$ comprising 8 studies published between 1991 and 2006 had a similar overall finding that combining psychosocial and pharmacologic interventions resulted in better outcomes than either no intervention or psychosocial interventions alone. When this was investigated further, there was an indication that "high intensity" smoking-cessation counseling was more effective than "low intensity" smoking-cessation counseling, although this was significant only when provided in combination with NRT. A similar limitation in that review was that motivation levels and the reporting of the COPD severity were inconsistent. Those authors concluded that the cessation success in this subject group is similar to that in non-COPD trials. A major barrier also was the lack of clarity over standardization of the smoking-cessation counseling for this group, as the intervention is often individualized to the patient's needs, so it is difficult to quantify and generalize these findings.

\section{Objectives}

The aim of this systematic review is to build on, update, and improve the findings of the earlier reviews by Wagena et $\mathrm{al}^{14}$ and Strassman et al, ${ }^{15}$ to identify the most effective stop smoking intervention approach for smokers with COPD. Changes were made to the search criteria, to identify relevant studies, such as terms to identify studies with a psychological element to the intervention. To improve the quality of the included studies, only those using a biochemical validation of smoking status were included.

\section{Methods}

\section{Criteria for Considering Studies for This Review}

Types of Studies. Randomized controlled trials or quasirandomized controlled trials were included.

Types of Subjects. Subjects were included in the studies if their diagnosis of COPD had been made as mild, moderate, or severe after assessment using guidelines outlined by the American Thoracic Society/European Respiratory Society Task Force ${ }^{16}$ or National Institute for Health and Care Excellence guidelines (2004). ${ }^{4}$ Studies that also comprised non-COPD diagnosed subjects in at least one comparison group were also included.

Types of Interventions. Studies investigating the effectiveness of smoking cessation interventions in smokers diagnosed with COPD with a minimum follow-up of 12 months after the conclusion of the intervention were included. Smoking status at follow-up was required to be validated using a biochemical marker, such as carbon monoxide in expired breath and/or saliva/urine cotinine. The intervention had to include a psychosocial and behavioral support element and a standard stop smoking medication such as NRT, bupropion, varenicline, or nortriptyline.

Types of Outcome Measures. The minimum outcome measure for inclusion was point prevalence quit status at 12 months, with a biochemical validation.

\section{Search Strategy to Identify Studies}

Electronic databases searched were MEDLINE, EMBASE, AMED, PsycINFO, DARE, Cochrane Library, and CINAHL. The initial search dates for all the databases were from January 2006 to January 2010. The references of the included studies were screened for additional papers, and the following journals were searched by Internet or by hand: Health Psychology, British Journal of Health Psychology, Journal of Health Psychology, Addiction, and Nicotine and Tobacco Research.

Search Terms. The following search terms used in the original systematic review by Wagena et $\mathrm{al}^{14}$ were used: COPD*, lung-diseases-obstructive*, emphysem*, bronchit*, tobacco, nicotine, smoking, smoking-cessation, tobacco-use-disorder, tobacco-smokeless, anti-smoking, quit*, stop*, cessat*, ceas*, abstin*, abstain*, control*, smok*, giv*, tobacco*.

The search terms used in the Strassman et al ${ }^{15}$ review followed broader categories, due to it being part of a larger review project on various treatments for COPD. With one exception, these terms had been included in the Wagena et $\mathrm{al}^{14}$ review; the one exception was "treatment outcome," which was considered by the authors to be too broad for the purposes of this review.

To identify relevant studies the following additional search terms were used: interven*, NRT, nicotine, bupropion, zyban, varenicline, champix, chantix, nortript*, antidepress*, counsel*, behavio*, CBT [cognitive behavioural therapy], and psycho*.

\section{Study Selection Criteria}

The search yielded 81 papers between January 2006 and January 2010. The abstracts were read and papers not meeting the full inclusion criteria were eliminated. This process resulted in 4 articles that were considered relevant for inclusion in this review.

In an attempt to expand the pool of studies for this review, the same database search was repeated from March 2002 (the end of the journal search for the Wagena et al ${ }^{14}$ review) to December 2005, to identify any additional papers that may have been missed from the Strassman et al ${ }^{15}$ review. This yielded one additional study, but it was excluded due to the lack of a 12-month follow-up. 


\section{Smoking Cessation Interventions for COPD: A Review of the Literature}

\section{Methodological Quality Assessment}

A quality assessment tool was designed for this review. In comparison to the previous 2 systematic reviews, ${ }^{14,15}$ this review did not use the full Delphi list ${ }^{17}$ to assess the quality of the studies. The Delphi list was developed as a minimum reference standard for randomized controlled trials on many different research topics, and, rather than replace, was intended to be used alongside other criteria lists. It was felt that the subject area of this review required an active role on the part of the subject in terms of behavioral support and a degree of control over their level of engagement with behavior change techniques. Consequently, it was decided that the elements of blinding of the care provider and the subject were not valid items, which, as Wagena et al $^{14}$ discussed, led to internal validity difficulties for their systematic review when comparing psychosocial interventions. To overcome this, a number of quality assessment points were added that also aimed to increase the validity of the studies selected.

The items in the quality assessment list and the scores attached to each were:

- Selection Bias: How were subjects randomized? Score: $2=$ computer randomized, $1=$ other randomization, $0=$ no explanation

- Comparable at Baseline: Did the groups appear comparable at baseline regarding the most important prognostic indicators (eg, numbers smoked, addiction level, age)? Score: $1=$ yes, $0=$ no

- Detection Bias: Was a biomarker used to validate selfreport at follow-up? Score: $2=$ used at $>1$ follow-up point, $1=$ used at 1 follow-up point, $0=$ not used/not clear

- Detection Bias: Was a biomarker used to validate selfreport at completion of intervention? Score: $3=>75 \%$, $2=50-75 \%, 1=<50 \%$

- Description of Intervention Procedure: Score: $3=$ stepby-step and psychological principles, $2=$ step-by-step, $1=$ some description, $0=$ barely any description

- Suitable Comparison Interventions (Medications): Score $2=$ comparable, $1=$ some comparison, $0=$ no comparison

- Description of Stage of Disease: Score: 1 = information given, $0=$ no information given

- Description of Type of Smoker (Light/Heavy): Score: $1=$ information given, $0=$ no information given

- Time Points of Follow-up: Score: $4=>12$ months post intervention, $3=6-12$ months post-intervention, $2=$ longer than end of intervention but $<6$ months postintervention, $1=$ end of intervention

- Sample Size Justification: Score: 1 = power calculation used, $0=$ power calculation not used

- Drop-Out Rate by Conclusion of Intervention: Score: $2=$ drop-out rate $\leq 25 \%, 1=$ drop-out rate $25-50 \%$, $0=$ drop-out rate $>50 \%$ or not known

- Intention-to-Treat Analysis (Non-Attendees at Completion Classed as Smokers): Score: $1=$ Yes, $0=$ No

The selected studies were reviewed independently by 2 reviewers (GA and RP-Y). Divergence of quality assessment scores was resolved by the reviewers meeting to discuss differences in ratings and agreeing on a score.

\section{Data Analysis}

Statistical data were analyzed using statistics software (SPSS 17, SPSS, Chicago, Illinois). The studies were heterogeneous with regards to:

- The study population and COPD severity: classified as mild ( $\mathrm{FEV}_{1} \geq 80 \%$ of predicted), moderate $\left(\mathrm{FEV}_{1} 50\right.$ $79 \%$ of predicted), or severe $\left(\mathrm{FEV}_{1} 30-49 \%\right.$ of predicted), as defined by the American Thoracic Society/ European Respiratory Society (2004) $)^{16}$

- Format of treatment (individual, group, or telephone) or behavioral/psychological support

- Use of pharmacologic treatments (no treatment, NRT, bupropion)

- Level of psychological assessment, including motivation measures

- Included study quality

Significance levels, effect sizes, odds ratios, and 95\% CIs were calculated where possible, and some statistical information in the primary research is reported where considered appropriate.

\section{Description of Studies}

Two studies were carried out in COPD out-patient clinics. ${ }^{18,19}$ One study was carried out in in-patients in an acute setting, with the control group in primary care settings. ${ }^{20}$ One study was carried out in a respiratory outpatient clinic, and the control group received no support after initial brief advice. ${ }^{21}$ All the studies had elements of psychological intervention. One study included group, individual, and telephone support, ${ }^{18}$ compared to a control group with individual and telephone support. One study offered group support, compared to a control group of unspecified usual care..$^{20}$ One study offered group support 


\section{Smoking Cessation Interventions for COPD: A Review of the Literature}

for both the experimental and control groups. ${ }^{19}$ And one study comprised 2 experimental arms: one with individual support and one with group support, plus a control group that received no additional support after initial brief advice. $^{21}$

The interventions were led by a range of healthcare professionals, including respiratory nurses, ${ }^{18}$ a smoking cessation nurse,$^{20}$ nurse specialists trained in asthma and COPD, a researcher trained in smoking cessation, ${ }^{19}$ and a respiratory nurse in the experimental groups..$^{21}$ In the latter study the control group received a brief intervention from a doctor. No information was available on the usual care provided in primary care..$^{20}$

The COPD severity was given for all subjects. All were diagnosed using the American Thoracic Society/European Respiratory Society ${ }^{16}$ or the National Institute for Health and Care Excellence (2004) ${ }^{4}$ classification as mild, moderate, or severe. One study comprised moderate and severe subjects, ${ }^{18} 2$ studies comprised subjects with 3 levels of diagnosis, ${ }^{20,21}$ and one study compared an experimental group comprising subjects with an average mild diagnosis to a control group of asymptomatic subjects. ${ }^{19}$

We concluded that one study gave little description of the intervention, ${ }^{18}$ one study gave a step-by-step description, ${ }^{20}$ and 2 studies gave a step-by-step description and the psychological principles. ${ }^{19,21}$

The smoking status of the subjects was measured using a number of tools in all the studies. The cigarettes smoked ranged from 17.5 to 24.1 per day. ${ }^{18,19,21}$ Nicotine dependence, measured using the Fagerstrom Test for Nicotine Dependence (1991), ranged from 4.7 to 4.8. ${ }^{20}$ Pack-years (calculated as number of cigarettes smoked per day $\times$ number of years smoked/20) ranged from 25 to 45.5.19-21

The characteristics of the studies can be found in the supplementary materials at http://www.rcjournal.com.

\section{Methodological Quality}

The quality scores of the studies range from 11 to 20 . One study used a computer-generated list of random numbers, ${ }^{21}$ one study was randomized according to other criteria (eg, the level of COPD), ${ }^{19}$ and 2 studies gave no explanation. ${ }^{19,20}$ Biomarker validation was used for $>75 \%$ of outcomes in 3 studies, using a range of measures: salivary cotinine test $(<20 \mathrm{ng} / \mathrm{mL}),{ }^{18}$ urinary cotinine with a cutoff set at $<25 \mathrm{ng} / \mathrm{mL},{ }^{19}$ and in one of these studies 2 measures were used: expired breath carbon monoxide with a cutoff at $\leq 10$ parts per million, and salivary cotinine with a cutoff level of $\leq 10 \mathrm{ng} / \mathrm{mL},{ }^{21}$ and less than $50 \%$ in one study using expired breath carbon monoxide monitoring with a cutoff set at $<8$ parts per million. ${ }^{20}$ Two studies ${ }^{19,21}$ used biomarkers on more than one follow-up, one study ${ }^{18}$ used biomarkers on one follow-up, and one study ${ }^{20}$ did not use a biomarker until the end of the study.
Only one study ${ }^{21}$ included a sample size justification or power analysis, yet failed to achieve the sample size required. Two studies had $>100$ subjects in each group, ${ }^{18,20}$ and 2 studies had $<40$ subjects per group. ${ }^{19,21}$ Two studies had a drop-out rate of $<25 \%, 19,20$ while 2 studies had drop-out rates of 25-50\%. ${ }^{18,21}$ Explanations for these dropouts were not consistently reported (see the supplementary materials at http://www.rcjournal.com).

\section{Results}

The primary aim of the 4 studies identified for this review was the effect of the interventions on smoking cessation. The supplementary materials at http://www. rcjournal.com show the outcomes of the studies included in this review. A meta-analysis was also conducted (see the supplementary materials at http://www.rcjournal.com).

All 4 measured abstinence 12 months post-intervention. Just one ${ }^{19}$ reported follow-up outcomes at points before this, while one reported follow-up outcomes 24 months after this point. The results for the 12 months will be reported and the results at 36 months follow-up will be discussed in the context of that study. The overall difference in the effectiveness of all the studies at 12 months between the experimental groups and the control groups was significant (chi-square test for heterogeneity chisquare $=39.7, P<.001$ ), with a total of $35.5 \%$ of subjects quitting smoking in the experimental groups, and $10 \%$ quitting smoking in the control groups (pooled randomeffects odds ratio $2.35,95 \%$ CI $0.25-21.74$ ). A by-studies comparison showed: in Sundblad et $\mathrm{al}^{20}$ there were similar numbers of subjects in the experimental and control groups ( $n=204$ and 219, respectively); in Christenhusz et al ${ }^{18}$ there were more subjects in the experimental group $(n=96$, compared to $n=67$ in the control group); in Willemse et $\mathrm{al}^{19}$ there were more subjects in the experimental group ( $n=38$, compared to $n=25$ in the control group).

\section{Different Psychosocial Interventions With Pharmacotherapy}

Christenhusz et al ${ }^{18}$ compared 2 interventions with subjects diagnosed with either moderate or severe COPD. One group received $595 \mathrm{~min}$ of support (high intensity), although the number of sessions was not stated. Delivered in group, individual, and telephone format, $100 \%$ were prescribed bupropion free of charge, and $6 \%$ also reported using NRT. The control group received 180 min (medium intensity) of individual and telephone support, although again it was not reported over how many sessions. In this study $28 \%$ used bupropion and $14 \%$ used NRT, although these were not free of charge. Respiratory nurses delivered both interventions. At 12 month follow-up 19\% were abstinent in the experimental group and 9\% were abstinent in 


\section{Smoking Cessation Interventions for COPD: A Review of the Literature}

the control group (odds ratio 2.35, 95\% CI 0.88-6.27). The high intensity group had a higher nicotine addiction score, measured by Fagerstrom (59\%, compared to $42 \%$ scoring $\geq 6$ ). Other independent variables measured included attitude to smoking cessation, self efficacy, and quality of life, although changes in these were nonsignificant $(P=.08)$ in the experimental group. For the control group a higher cotinine value at baseline led the authors to indicate that each rise of $100 \mathrm{ng} / \mathrm{mL}$ doubled the likelihood of quitting and that a positive attitude toward stopping success increased by 12 times (both variables $P=$ .003). However, a large confidence interval of the odds ratio was reported (odds ratio 22.52, 95\% CI 1.55-327.97), which indicates low precision in this estimate.

Sundblad et $\mathrm{al}^{20}$ compared a high intensity in-patient intervention with usual care provided in primary care health centers. A smoking cessation nurse delivered the experimental arm for 1 hour per day in groups of 4-8 people over 11 days, as part of a wider lifestyle intervention with additional input from a doctor, a physiotherapist, a dietitian, a laboratory technician, a psychologist, an occupational therapist, and a nurse. NRT was recommended, and was used by $28 \%$ in the experimental group and $14 \%$ in the control group. There were also regular follow-up telephone calls for $2-3$ months after discharge, and the subject and his or her spouse then returned for 2-4 days as inpatients, followed by additional telephone support follow-up until 12 months post-intervention. No information was given on the usual care offered for the control group, although just 20\% (46) accessed this. At 1 year, point prevalence abstinence (in the previous 6 months) was significant (chi-square $=105.2, P<.001$ ), with $52 \%$ in the intervention group and 7\% in the control stopping (odds ratio $14.71,95 \%$ CI 8.14-26.59). At 3 years these figures had reduced to $38 \%$ and $10 \%$, respectively, but the treatment difference was still significant (chi-square $=44.0$, $P<.001)$. There was no difference in outcome based on the severity of the disease. There were no differences reported in nicotine dependence between the groups at baseline, although the authors acknowledged that which part of the intervention was effective is difficult to deduce, given the mixed use of pharmacotherapy and the extensive format of the wider intervention.

Wilson et $\mathrm{al}^{21}$ compared a high intensity individual intervention and a high intensity group intervention (5 sessions of 1 hour each) provided by one respiratory nurse and 2 respiratory nurses, respectively, (including an initial 5-10 min brief intervention) with an individual usual care, brief intervention (5-10 min) only, delivered by a doctor, in an out-patient department. The experimental arms also offered 12 weeks of NRT, while no information is available for the control group. No quitters were reported in either group at 12 month follow-up, so no effect size was calculated. However, it was reported that all groups had a significant reduction in nicotine addiction over this period: $P=.006$ between the control and group intervention, and $P=.03$ between the control and individual support.

\section{Comparison By Disease Presence}

Willemse et al ${ }^{19}$ studied a group intervention $(8-10$ per group) in an out-patient setting. The experimental group had been diagnosed with mild COPD and the control group was asymptomatic. A respiratory nurse specialist and a researcher trained in smoking cessation delivered the same intervention. Nine 2-hour sessions were initially offered over 6 weeks. Based on programs developed in the Netherlands, they incorporated cognitive behavioral therapy techniques to build motivation, and self-efficacy and coping skills. Additional telephone support was offered in between sessions, and 6 additional group sessions were offered, ad hoc, over the remaining time to 12 months, provided by the same facilitators. No pharmacotherapy was recommended. At 12 months, continual abstinence was significant (chi-square $=4.05, P<.05$ ), although with $42 \%$ in the intervention group and $68 \%$ in the control group, so the control group was more successful (odds ratio $0.34,95 \%$ CI $0.12-0.99$ ).

\section{Discussion}

The heterogeneity between studies with respect to populations and interventions, combined with few studies with relatively few subjects, meant that any estimation of an effect size was bound to be uncertain. So, although the intervention looked promising, it is not possible on current evidence to quantify the effect or confirm that the promising results observed are not the result of chance. Further, odds ratios, while widespread, are problematic as effect size measures when the frequency of the outcome is high.

\section{Disease Severity}

Subjects with different COPD severity within and between each study make clear conclusions difficult. Therefore, as was also recommended in the Wagena et $\mathrm{al}^{22}$ review, sub-analysis would be preferable wherever possible (eg, as in Sundblad et $\mathrm{al}^{20}$ ).

The time since diagnosis was not reported, which could impact the subject's perception of their smoking behavior and its impact on their illness and development of coping strategies to live with and manage exacerbations. A future study could therefore investigate the effect of time from diagnosis on smoking cessation outcomes as another inclusion criterion.

It is important to highlight that, given that one of the studies contained a control group with non-COPD patients, the estimated effect sizes may not be generalizable. 


\section{Smoking Cessation Interventions for COPD: A Review of the Literature}

\section{Levels of Addiction and Intensity of Intervention}

Consistent reporting according to disease severity would help clarify the effects of level of addiction and intensity of interventions, as illustrated by 2 of the studies. Higher cotinine levels resulted in a better outcome, contrary to popular assumptions regarding level of addiction and successful quitting, ${ }^{18}$ leading to the suggestion that the higher intensity compensates for higher addiction in one group, while a higher favorable attitude score to cessation found in the less intense control could compensate for the higher addiction. One hypothesis by the authors is that smokers with a high cotinine level may have a more internal motivation, as they may experience worse symptoms, whereas those with a lower cotinine level may have a more external, less stable motivation (eg, being advised to stop by a practitioner). In another study ${ }^{19}$ the intervention group had mild COPD but higher pack-years than the control group. The high quit rate in both groups was thought to be possibly due to high contact time and intense baseline and follow-up measurements, with frequent and intensive motivational support being proposed to account for the high cessation rate, suggesting a flexible approach to cessation, with interventions designed depending on initial findings of screening.

\section{Effect of Healthcare Professional}

As found by the Strassman et al ${ }^{15}$ review, there were a number of points in the studies in this review when the person delivering the intervention could have affected the intervention, for example during ad hoc telephone support. This additional support could offer an opportunity for relapse prevention, but its lack of measurement can make it more difficult to generalize the findings.

\section{Attendance}

Poor attendance was an issue for usual care conditions. ${ }^{20,21}$ Wilson et $\mathrm{al}^{21}$ recommend that research investigates more acceptable interventions or that their study is repeated in a community setting. In comparison, Willemse et $\mathrm{a}^{19}$ found that a medical setting may have had an effect on their study, hypothesizing that the medical research environment may have made the subjects feel more responsible for the outcome. This may have made subjects more serious toward the intervention, questioning the balance between "medicalizing" an intervention and ease of access in a community setting. The effect of exacerbations during the studies was not measured. These could affect attendance and adherence to the use of stop smoking medications, and it could be hypothesized that they may be more likely to attend a medical setting during such epi- sodes. Additional information on the health of the subjects during the course of the studies would be useful.

\section{Social Support and Motivation}

Social circumstances should also be considered. Wilson et $\mathrm{al}^{21}$ reported that $42 \%$ lived with a smoker, and this may have affected their perception of the importance of cessation, or they may not have received the peer support that could have effected a successful outcome. Similarly, motivation of subjects to take part was not measured consistently and varied from a measured "stage of change" identification to being estimated by their attendance at an information session and the signing of an informed consent form as a proxy measure for motivation. ${ }^{18,20}$ Poorer outcomes could possibly be a function of the subject selection; at recruitment a number of the studies indicated that a high proportion of the subjects had already quit smoking, leaving a potential sub-group who could be described as "recalcitrant" smokers.

\section{Follow-up and Biomarkers}

It is acknowledged that a large percentage of the general population relapse within the first 12 months post-quitting. For a complex group such as COPD patients this could therefore be considered too long a period as a measure and also to indicate that the level of initial support, or follow-up relapse prevention support, is not sufficient, as the aim of stop smoking interventions is to provide smokers with the skills to maintain abstinence.

Three out of the 4 studies used continuous abstinence as a measure, while one study used a long duration point prevalence marker of 6 months. Van der Meer et $\mathrm{al}^{23}$ recognized that, based on the findings of Velicer et al, ${ }^{24} \mathrm{a}$ combination of continuous abstinence and point prevalence measures should be used to assess outcomes to reach a clearer picture of the quitting process. Although not used by the studies under review, this would illustrate the dynamic nature of smoking cessation. Point prevalence would raise quit rates in the short-term, increasing self-efficacy in that subjects can stop rather than being labeled a "failure" for not achieving continuous abstinence status, while continuous abstinence would help to identify better assessment of the longer-term effects of an intervention.

\section{Conclusions}

The primary aim of the studies identified in this review was to establish the effectiveness of psychosocial interventions, with the effect of pharmacotherapy being a secondary outcome. It has been difficult to establish the most effective approach to smoking cessation to take with a COPD population. Despite stricter inclusion criteria, there 


\section{Smoking Cessation Interventions for COPD: A Review of the Literature}

were still many differences in the composition and level of intensity of interventions and the subjects themselves. The outcome of quit/not quit does not appear to allow sufficient scope for measuring the effect of the constituent parts of a psychosocial intervention. Without this better measurement, biomedical approaches may appear more effective; for example, Bittoun ${ }^{25}$ found that more NRT use without explicit psychosocial intervention can be very effective at achieving a non-smoking status for this group. In this review there was no consistent use of or access to stop smoking pharmacotherapies, which could obscure the effects of the psychological components of the interventions under review.

However, consistent with Strassman et al, ${ }^{15}$ a combination of pharmacotherapy and psychosocial support appears to be the best approach to take to stopping smoking, although a lengthy intervention does not necessarily result in better outcomes. Locating the interventions in a medical setting does appear to have a positive impact on initiation of a treatment, and may be a method of improving ongoing engagement with support services, in comparison to standard usual care services in primary care or community settings.

This review highlights the challenge for future research to develop more tailored interventions to reduce smoking prevalence in a chronic clinical population where a range of behavior changes is essential to managing their health and improving well-being.

\section{REFERENCES}

1. World Health Organization. Chronic respiratory diseases: chronic obstructive pulmonary disease (COPD). http://www.who.int/respira tory/COPD/en. Accessed August 21, 2013.

2. Mannino DM. COPD: epidemiology, prevalence, morbidity and mortality, and disease heterogeneity. Chest 2002;121(5 Suppl):121S$126 \mathrm{~S}$.

3. United Kingdom National Health Service. Chronic obstructive pulmonary disease: introduction. http://www.nhs.uk/Conditions/Chronicobstructive-pulmonary-disease/Pages/Introduction.aspx. Accessed August 21, 2013.

4. National Institute for Health and Clinical Excellence. Quick reference guide: chronic obstructive pulmonary disease: management of chronic pulmonary disease in adults in primary and secondary care. 2004. http://www.nice.org.uk/CG012quickrefguide. Accessed August 21, 2013.

5. Department of Health. NHS Stop Smoking Services: service and monitoring guidance 2009/10. London: The Stationery Office; 2009.

6. Jiménez-Ruiz CA, Masa F, Miravitlles M, Gabriel R, Viejo JL, Villasante C, Sobradillo V. Smoking characteristics. Differences in attitudes and dependence between healthy smokers and smokers with COPD. Chest 2001;119(5):1365-1370.

7. Walters N, Coleman T. Comparison of the smoking behaviour and attitudes of smokers who attribute respiratory symptoms to smoking with those who do not. Br J Gen Pract 2002;52(475):132-134.
8. Hill K, Geist R, Goldstein RS, Lacasse Y. Anxiety and depression in end-stage COPD. Eur Respir J 2008;31(3):667-677.

9. Bourbeau J, Bartlett SJ. Patient adherence in COPD. Thorax 2008; 63(7):831-838.

10. Coventry PA, Gellatly JL. Improving outcomes for COPD patients with mild-to-moderate anxiety and depression: a systematic review of cognitive behavioural therapy. Br J Health Psychol 2008;13(3): 381-400.

11. Stage KB, Middelboe T, Stage TB, Sorensen CH. Depression in COPD: Management and quality of life considerations. Int J COPD 2006;(3):315-320.

12. Wedzicha JA, Calverley PM, Seemungal TA, Hagan G, Ansari Z, Stockley RA. The prevention of chronic obstructive pulmonary disease exacerbations by salmeterol/fluticasone propionate or tiotropium bromide. Am J Respir Crit Care 2008;177(1):19-26.

13. Calverley PM, Anderson JA, Celli B, Ferguson GT, Jenkins C, Jones PW. Salmeterol and fluticasone propionate and survival in chronic obstructive pulmonary disease. N Engl J Med 2007;356(8):775-89.

14. Wagena EJ, van der Meer RM, Ostelo RJWG, Jacobs JE, Schayck $\mathrm{CP}$. The efficacy of smoking cessation strategies in people with chronic obstructive pulmonary disease: results from a systematic review. Respir Med 2004;98(9):805-815.

15. Strassman R, Bausch B, Spaar A, Kleijnen J, Braendli O, Puhan MA. Smoking cessation interventions in COPD: a network meta-analysis of randomized trials. Eur Respir J 2009;34(3):634-640.

16. American Thoracic Society. COPD guidelines. http://www.thoracic. org/go/COPD. Accessed August 21, 2013.

17. Verhagen AP, de Vet HC, de Bie RA, Kessels AG, Boers M, Bouter LM, Knipschild AG. The Delphi list: a criteria list for quality assessment of random clinical trials for conduction systematic reviews developed by Delphi consensus. J Clin Epidemiol 1998;51(12):12351241.

18. Christenhusz L, Pieterse M, Seydel E, van de Palen J. Prospective determinants of smoking cessation in COPD patients within a high intensity or a brief counseling intervention. Patient Educ Couns 2007; 66(2):162-166.

19. Willemse B, Lesman-Leegte I, Timens W, Postma D, ten Hacken N. High cessation rates of cigarette smoking in subjects with and without COPD. Chest 2005;128(5):3685-3687.

20. Sundblad BM, Larsson K, Nathell L. High rate of smoking abstinence in COPD patients: Smoking cessation by hospitalization. Nicotine Tob Res 2008;10(5):883-890.

21. Wilson JS, Fitzsimons D, Bradbury I, Elborn JS. Does additional support by nurses enhance the effect of a brief smoking cessation intervention in people with moderate to severe chronic obstructive pulmonary disease? A randomized controlled trial. Int J Nurs Stud 2008;45(4):508-517.

22. Wagena EJ, de Graaf L, Chavannes NH, van Grootheest AC, van Schayck CP. [Uneasiness about the safety of bupropion as an aid to smoking cessation unjustified]. J Ned Tijdschr Geneeskd 2001; 145(31):1489-1492. Article in Dutch.

23. Van der Meer RM, Wagena EJ, Ostelo RW, Jacobs JE, van Schayck CP. Smoking cessation for chronic obstructive pulmonary disease. Cochrane Database Syst Rev 2003;(2):CD002999.

24. Velicer WF, Prochaska JO, Rossi JS, Snow MG. Assessing outcome in smoking cessation studies. Psychol Bull 1992;111(1):23-41.

25. Bittoun R. A combination nicotine replacement therapy (NRT) algorithm for hard-to-treat smokers. J Smok Cessat 2006;1(1):3-6. 